OPEN ACCESS

Edited by:

Hamid R. Sohrabi,

Macquarie University, Australia

Reviewed by:

Rodolfo Gabriel Gatto,

University of Illinois at Chicago,

United States

Aurel Popa-Wagner,

University of Medicine Essen,

Germany

*Correspondence:

Andreas Hermann

andreas.hermann@

uniklinikum-dresden.de

Specialty section:

This article was submitted to Neurodegeneration, a section of the journal

Frontiers in Neurology

Received: 23 November 2017 Accepted: 05 February 2018

Published: 26 February 2018

Citation:

Günther R, Schrempf W, Hähner A,

Hummel T, Wolz M, Storch A and

Hermann A (2018) Impairment in

Respiratory Function Contributes to

Olfactory Impairment in Amyotrophic

Lateral Sclerosis.

Front. Neurol. 9:79.

doi: 10.3389/fneur.2018.00079

\section{Impairment in Respiratory Function Contributes to Olfactory Impairment in Amyotrophic Lateral Sclerosis}

\author{
René Günther ${ }^{1,2}$, Wiebke Schrempf ${ }^{1}$, Antje Hähner ${ }^{3}$, Thomas Hummel ${ }^{3}$, Martin Wolz ${ }^{4}$, \\ Alexander Storch ${ }^{2,5,6}$ and Andreas Hermann ${ }^{1,2 *}$ \\ 'Division for Neurodegenerative Diseases, Department of Neurology, Technische Universität Dresden, Dresden, Germany, \\ ${ }^{2}$ German Center for Neurodegenerative Diseases (DZNE) Dresden, Dresden, Germany, ${ }^{3}$ Smell \& Taste Clinic, Department \\ of Otorhinolaryngology, Technische Universität Dresden, Dresden, Germany, ${ }^{4}$ Department of Neurology, Elblandkliniken \\ Meissen, Meissen, Germany, ${ }^{5}$ Department of Neurology, University of Rostock, Rostock, Germany, ${ }^{6}$ German Center for \\ Neurodegenerative Diseases (DZNE) Rostock, Rostock, Germany
}

Background: Nonmotor symptoms are very common in neurodegenerative diseases. In patients suffering from amyotrophic lateral sclerosis (ALS), olfactory dysfunction was first reported more than 20 years ago; however, its pathophysiological correlates and further implications remain elusive.

Methods: In this so far largest case-control study, we analyzed olfactory performance with the "Sniffin' Sticks," a validated olfactory testing kit used in clinical routine. This test kit was designed to investigate different qualities of olfaction including odor threshold, odor discrimination, and odor identification.

Results: ALS patients were mildly but significantly impaired in TDI score, the composite of the three subtests (ALS $27.7 \pm 7.9$, Controls $32.3 \pm 5.8$ ). In contrast to Parkinson's disease, ALS patients did not show impaired performance in the suprathreshold tests identification and discrimination. However, the odor threshold was markedly decreased (ALS $6.0 \pm 3.4$, Controls $8.77 \pm 3.6$ ). This pattern of olfactory loss resembles sinonasal diseases, where olfactory dysfunction results from impeded odorant transmission to the olfactory cleft. The evaluation of medical history and clinical data of ALS patients showed that patients with perception of dyspnea (TDI $25.7 \pm 8.0$ ) performed significantly worse in olfactory testing compared to those who did not (TDI $30.0 \pm 7.4$ ). In line with that, we found that in patients with preserved respiratory function (vital capacity $>70 \%$ of index value), olfactory performance did not differ from healthy controls.

Conclusion: These findings suggest that the mild impairment of olfaction in patients suffering from ALS should at least partly be considered as a consequence of impaired respiratory function, and odor threshold might be a marker of respiratory dysfunction in ALS.

Keywords: amyotrophic lateral sclerosis, olfaction, hyposmia, sniffing, vital capacity, respiratory function 


\section{INTRODUCTION}

Amyotrophic lateral sclerosis (ALS) and its variants, primary lateral sclerosis and progressive muscular atrophy, are fatal neurodegenerative disorders with progressive degeneration of motor neurons and their axons (1-3). For many neurodegenerative diseases, it is well known that the pathology is not limited to the initially affected cell populations. Instead, disease spreading and involvement of other nonmotor regions in the brain seem to occur (4). Corresponding nonmotor symptoms like gastrointestinal, autonomic, neuropsychiatric, and sleep disorders are well known in these diseases. Treatments of such nonmotor symptoms (NMSs) are fundamental elements in a modern and comprehensive health care for patients suffering from neurodegenerative diseases. A wide range of NMS are known from Parkinson's disease (PD) $(5,6)$. Frontotemporal impairment and memory loss are commonly known as "nonmotor" involvements in patients with ALS (7-10). Besides that, multiple other extramotor symptoms in ALS have been reported in the last years (11, 12), for example, autonomic dysfunction (13-17), sensory (18), and extrapyramidal symptoms $(19,20)$. Neuroanatomical studies in sporadic forms of ALS revealed a disease stage-dependent distribution of phosphoTDP-43 in different, later also nonmotor, brain regions $(3,21,22)$. In addition, substantia nigra hyperechogenicity, also considered as prodromal marker for $\mathrm{PD}$, was found in ALS patients in similar frequency to PD (23).

We recently assessed the self-rating NMS questionnaire (NMSQuest) in patients suffering from ALS and its variants. Although the absolute frequency of NMS was not as high as in PD patients, ALS patients complained about impaired taste and/or smell significantly more often than healthy controls (5, 24). Hyposmia is one of the most common NMS in PD and can antedate classical motor symptoms by several years $(25,26)$. The olfactory system is one of the structures primarily affected in PD and is discussed as the starting point of disease propagation (27, 28). Olfaction impairment in ALS is not yet studied intensively. However, hyposmia was first reported in small populations of idiopathic ALS and later in Guamanian Chamorro ALS patients by means of the University of Pennsylvania Smell Identification Test (UPSIT) and cultural-adapted versions (29-32). A more comprehensive trial using the UPSIT in 58 ALS patients proved a slightly decreased overall smell identification compared to controls, but only patients with bulbar symptoms showed a significant impairment (33). More recently, a small prospective study $(N=26)$

\footnotetext{
Abbreviations: ALS, amyotrophic lateral sclerosis; ALSDys, patients with dyspnea; ALSnoDys, patients without dyspnea; ALSppFVC $<70$, patients with FVC below $70 \%$ of their predicted healthy index value; ALSppFVC $>70$, patients with FVC above $70 \%$ of their predicted healthy index value; ALSFRS-R, revised ALSFunctional-Rating Scale; BMI, body mass index; MWU, Mann-Whitney U-Test; NMS, Nonmotor symptoms; Bulb, patients with bulbar symptoms; ClassALS, classical ALS with both UMNS and LMNS; D, odor discrimination; FVC, forced vital capacity; I, odor identification; PD, Parkinson's disease; KW, Kruskal-WallisANOVA followed by median test dialog; LMNS, lower motoneuron symptoms; MN, motoneuron; NoBulb, patients without bulbar symptoms; ppFVC, FVC in percentage of predicted healthy index value; T, odor threshold; TDI, composite of T, I, D; UMNS, upper motoneuron symptoms; UPSIT, University of Pennsylvania Smell Identification Test; $\chi^{2}$, chi-squared test.
}

using the 12 odors Sniffin Sticks test, a simple screening test, did not show any differences between ALS and matched controls with the limitation of a relatively small sample size (34). Another small study showed that hyposmia is common in a subgroup of ALS patients with cognitive impairment (35). Interestingly, a recent neuropathology study on postmortem samples of ALS patients showed that TDP-43-positive inclusion bodies could also be observed in the olfactory system (36).

The quality of olfactory function not only depends on the transmission and processing of odor information in the brain. Smelling is also dependent on various nonolfactory elements of the nose like airflow and mucociliar function. Respiration is one the main factors responsible for the active transport of stimulatory molecules to the sensory epithelia of the nasal cavity. The vast majority of olfactory dysfunction is caused by sinonasal diseases (37) including inflammatory diseases and respiratory dysfunction. Olfaction needs complex sensory-motor integration. Sniffing is the motor component which transports odorous substances to the olfactory receptors in the olfactory cleft, and olfactory performance depends on sniffing performance (38). Interestingly, hyposmia in $\mathrm{PD}$ is not exclusively caused by neurodegeneration; it is also partly caused by the impairment of sniffing, e.g., sniff airflow rate and sniff volume $(39,40)$. Sniff volume is one of the most important parameters for maximizing olfactory performance (41). Denervation of respiratory muscles and consecutive respiratory dysfunction with a decline of vital capacity is an obligatory phenomenon in ALS. Another sensitive parameter to predict respiratory dysfunction is the sniff nasal inspiratory pressure, and in a recent study, this parameter was shown to be more sensitive than vital capacity to predict the need for noninvasive ventilation. Sniff nasal inspiratory pressure declines very early and is much stronger than vital capacity in ALS (42).

The aim of this study was to screen for impairments in olfactory function, to assess the type of olfactory dysfunction, and to identify contributing factors in the so far largest case-control study concerning olfactory function in patients suffering from ALS.

\section{MATERIALS AND METHODS}

\section{Patients}

Patients with definite, probable, or possible ALS, according to the revised El Escorial criteria $(43,44)$, as well as patients with primary lateral sclerosis and progressive muscular atrophy, were recruited from October 2011 to May 2017 at the Department of Neurology of the University Hospital Dresden, Technische Universität Dresden. Patients suffering from spinal muscular atrophy, spinal bulbar muscular atrophy (Kennedy syndrome), frontotemporal dementia overlap syndromes, and daily life affecting or clinically relevant dementia were not included. Patients with the stated diagnosis of pulmonary diseases or with diseases of the upper airway were not included; however, a diagnostic checkup for the presence of other pulmonary or upper airway diseases was not performed. In addition, an age- and sex-matched control group of healthy individuals was tested with the standardized 'Sniffin' Sticks" test kit. The study was approved by the institutional review 
board at the Technische Universität Dresden, and patients gave their informed consent.

\section{Assessments}

Olfactory testing was performed using the "Sniffin' Sticks" test kit $(45,46)$ which involves tests for odor threshold ("T"), odor discrimination ("D"), and odor identification ("I"). Odor identification was assessed for 16 common odors (orange, leather, cinnamon, menthol, banana, lemon, licorice, garlic, coffee, apple, pineapple, rose, fish, anise, clove, and turpentine) using a multiple forced choice design, namely subjects identify odors by selecting the best label from a list of four descriptors. Results of the three subtests are presented as a composite score (TDI) ("TDI score") (range: 1-48), which is the sum of the results obtained for threshold (range: 1-16), discrimination (range: 0-16), and identification (range: 0-16) measures (45).

We additionally recorded age, gender, upper and lower motoneuron symptoms (UMNS, LMNS), bulbar symptoms, disease subtype at onset, forced vital capacity (FVC), FVC in percentage of predicted healthy index value (ppFVC) - which included adjustment for age, gender, and body height based on large databases of normative data $(47,48)$, body weight, body height, body mass index (BMI), disease duration (time between the first symptom and the date of test); the revised ALS-FunctionalRating Scale (ALSFRS-R); ALSFRS-R respiratory subscore, ALSFRS-R slope (the decline of ALSFRS-R from onset to the date of olfactory testing), complaining about dyspnea, tracheostomy, gastrostomy, noninvasive and invasive ventilation, REM sleep behavior disorder screening questionnaire (RBD-SQ), and NMSQuest.

\section{Statistical Analysis}

As the samples were not normally distributed by calculation with Shapiro-Wilk test, statistical comparisons of data between groups were made using the non-parametric Mann-Whitney U-test (MWU) for comparisons of two groups and the non-parametric Kruskal-Wallis (KW)-ANOVA followed by median test dialog for multiple group comparisons. The chi-squared test $\left(\chi^{2}\right)$ was carried out for a comparison of gender distribution between patients and controls. Spearman rank correlation coefficients were used to examine correlations between data of the "Sniffin' Sticks" test kit and demographic as well as clinical data in the ALS group with a correlation coefficient of rho $<0.3$ considered as a weak, rho $=0.3-0.59$ a moderate, and rho $\geq 0.6$ a strong correlation. Data were analyzed using the software programs SPSS 21.0 (SPSS Inc., Chicago, IL, USA) and Statistica 13.2 [StatSoft (Europe) $\mathrm{GmbH}$, Hamburg, Germany]. If not mentioned otherwise, all data are displayed as means \pm standard deviation. Significance level was set at $p<0.05$.

\section{RESULTS}

“Sniffin' Sticks" test kit data from 94 MND patients and 81 healthy controls were analyzed and compared. Demographic and clinical characteristics of the study populations are shown in Table $\mathbf{1 .}$ The two groups did not differ significantly regarding age (MWU, $p=0.20)$ or $\operatorname{sex}\left(\chi^{2}, p=0.06\right)$.
TABLE 1 | Demographic and clinical characteristics of study populations.

\begin{tabular}{lcc}
\hline & Control group & ALS group \\
\hline Total number & 81 & 94 \\
Ratio of female in \% & 64.2 & 50.0 \\
Age in years & $62.7 \pm 15.9$ & $64.5 \pm 10.2$ \\
Disease duration in years & - & $2.4 \pm 2.4$ \\
ALSFRS-R (range) & - & $35.2(18-48)$ \\
$\quad$ Respiratory subscore & & $10.7(4-12)$ \\
Subtype at onset $(n)$ & - & Spinal $(64)$ \\
& & Bulbar $(21)$ \\
& 0 & 78.7 \\
UMNS in \% & 0 & 90.4 \\
LMNS in \% & 0 & 52.7 \\
Bulbar symptoms in \% & 0 & 0 \\
Tracheostomy $(n)$ & 0 & 3 \\
Gastrostomy $(n)$ & 0 & 0 \\
Invasive ventilation $(n)$ & 0 & 7 \\
Noninvasive ventilation $(n)$ & 0 & 54.9 \\
Dyspnea in \% & - & $1984.1 \pm 973.9$ \\
ppFVC in \% & - & $26.3 \pm 4.5$ \\
FVC in ml & - & Primal sclerosis $(9)$ \\
BMl & &
\end{tabular}

\section{Mild Impairment of Olfactory Function in Patients Suffering from ALS}

Patients suffering from ALS performed worse in comparison to healthy controls regarding total score "TDI" (ALS 27.7 \pm 7.9 , Controls 32.3 $\pm 5.8 ; p<0.0001$ ) and threshold "T" (ALS 6.0 \pm 3.4 , controls $8.77 \pm 3.6 ; p<0.0001$ ) (Figures 1A,B). This means a loss of approximately $24 \%$ in "TDI" and $32 \%$ in "T". Discrimination score "D" (ALS 10.6 \pm 3.3 , Controls $11.5 \pm 2.1 ; p=0.06$ ) and identification score "I" (ALS 11.3 \pm 3.2 , Controls $12.0 \pm 2.2 ; p=0.32$ ) were not significantly different to controls (Figures 1C,D). As demonstrated before on a large study group, people older than 55 years do not have sex-dependent differences in “TDI" score (46). Our study group consisted of people with a mean age over 60 years (Table 1), and we also did not find significant sex-dependent differences (TDI control group: males $32.9 \pm 6.0$, females $32.0 \pm 5.7$; $p=0.41$; TDI ALS group: males $28.4 \pm 7.6$, females $27.1 \pm 8.2$; $p=0.26$; also no differences between $\mathrm{T}, \mathrm{I}$, and $\mathrm{D}$ data shown).

\section{Motoneuron (MN) Disease Subtype Did Not Show Particular Impairment of Olfactory Performance}

To find further influencing clinical parameters, we compared subgroups defined to clinically affected $\mathrm{MN}$ type at the date of test, labeled as only LMNS, only UMNS, and the classical ALS with affection of both MNs [classical ALS with both UMNS and LMNS (ClassALS)]. The three groups did not differ significantly for "TDI," “T," "D," "I" (“TDI": LMNS 27.5 \pm 7.0, UMNS $30.8 \pm 8.2$, ClassALS $27.4 \pm 8.1, p=0.65 ; \mathrm{T}, \mathrm{D}$, and I data not shown) (Figure 2).

\section{Mild Impairment of Olfactory Performance Occurs in Patients without Bulbar Symptoms (NoBulb)}

Previous published work hypothesized that hyposmia in patients suffering from ALS is only present in patients with bulbar 

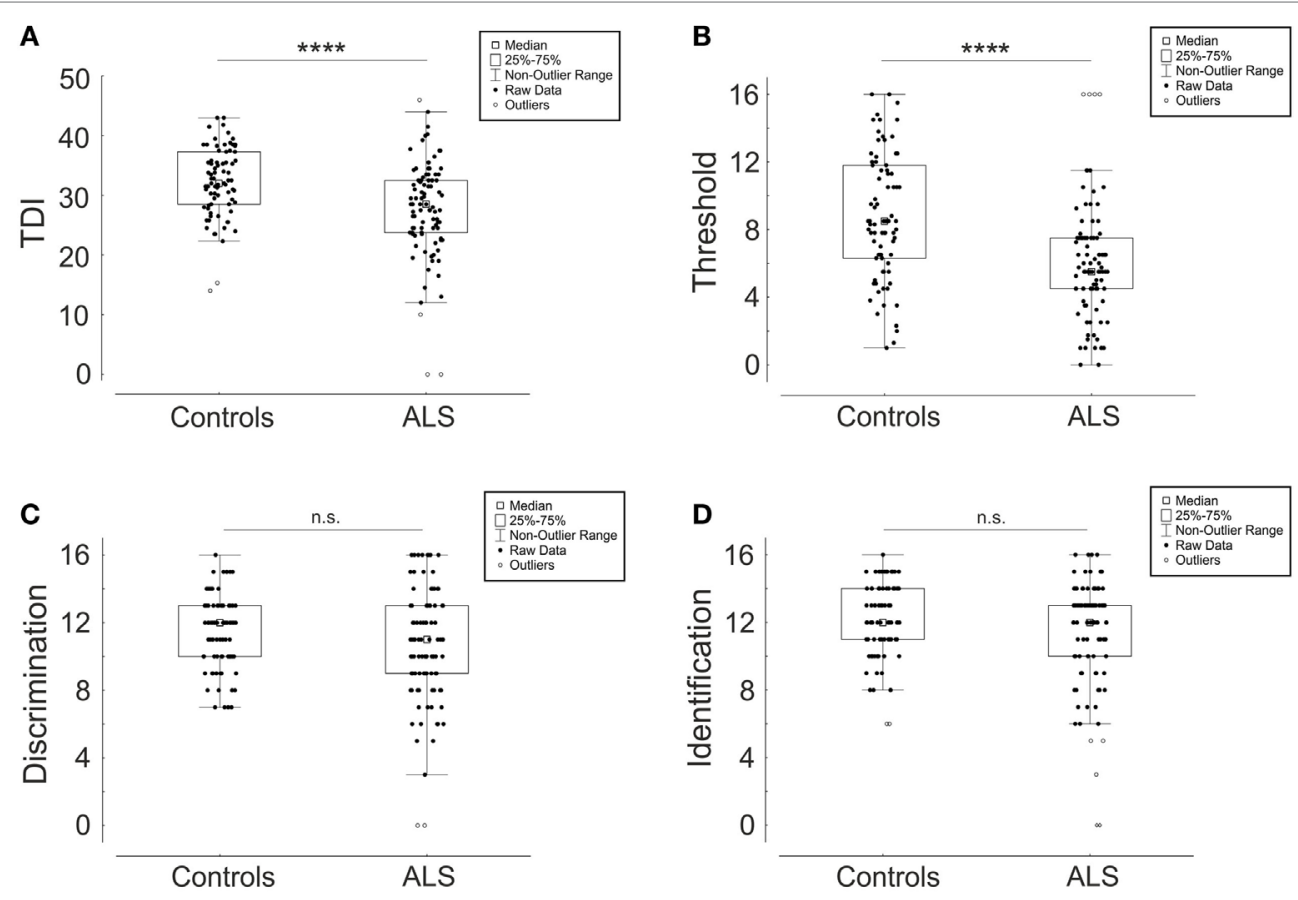

FIGURE 1 | Comparison of "Sniffin Sticks" test results between amyotrophic lateral sclerosis (ALS) patients and healthy controls depicted as box plots with single values (dots). TDI score (A) is a composite of the three subtests threshold "T" (B), discrimination "D" (C), and identification "I" (D). ${ }^{\star \star \star \star} p<0.0001$, not significant (n.s.).

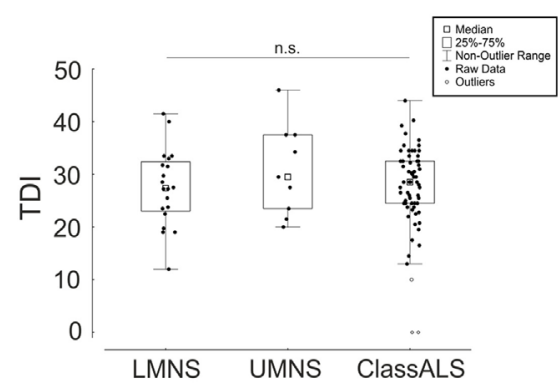

FIGURE 2 | Comparison of the composite of the three subtests (TDI) between amyotrophic lateral sclerosis (ALS) patients with prominent lower motoneuron symptoms (LMNS), upper motoneuron symptoms (UMNS), and classical ALS with both symptoms (ClassALS). Box plots with single values (dots). Not significant (n.s.).

symptoms (Bulb) (33). However, in our analysis, alterations occur in NoBulb for “TDI" (NoBulb 29.6 \pm 6.2 , Bulb 26.0 \pm 9.1, Controls $32.3 \pm 5.8, p<0.00001$ for Bulb vs Controls and $p<0.05$ for NoBulb vs Controls) and "T" (NoBulb $6.4 \pm 3.5$, Bulb $5.6 \pm 3.3$, Controls 8.77 $\pm 3.6, p<0.00001$ for Bulb vs Controls and $p<0.001$ for NoBulb vs Controls). "D” (NoBulb $11.2 \pm 2.6$, Bulb $10.0 \pm 3.8$, Controls $11.5 \pm 2.1, p=0.07$ ) and "I" (NoBulb $12.3 \pm 2.2$, Bulb $10.5 \pm 3.7$, Controls $12.0 \pm 2.2, p=0.08$ for Bulb vs Controls and $p=1.0$ for NoBulb vs Controls) were not altered in both patients with and without bulbar symptoms. Although NoBulb and Bulb did not differ significantly in "TDI" and "T", there is a propensity in the mean values toward worse performance in Bulb. In addition, a significant difference to less correct "I" $(p<0.05)$ in Bulb compared to that in NoBulb was calculated. But focused on statistical comparison to Controls, there is still not a relevant reduction of "I" performance in Bulb (see above). Taken together, bulbar palsy alone did not completely explain the worse Sniffin Sticks test results in patients suffering from ALS (Figure 3A).

\section{Patients Complained about Dyspnea Had Worse Olfactory Function}

Olfactory impairment was evident in patients suffering from ALS, but in our results, the pattern of olfactory loss resembles sinonasal diseases, where olfactory dysfunction results from impeded odorant transmission to the olfactory cleft rather than a pattern of a neurodegenerative disease etiology like PD (49). Thus, we hypothesized that the obligate decline in the respiratory function of ALS patients is a contributing factor for the impairment in olfactory function. Therefore, we investigated the natural history of patients and compared patients who complained about dyspnea (ALSDys, $N=50$ ) to patients who did not [ALS patients without dyspnea (ALSnoDys), $N=41$ ]. Complaining of dyspnea was either recorded from the patients' history and/ or from ALSFRS-R respiratory subscore. "TDI" of ALSnoDys assimilates to "TDI" of control group and statistically changed into not significantly different. By contrast, “TDI” of ALSDys group performed worse than the baseline value of the overall ALS cohort and was significantly impaired in comparison to Controls and to ALSnoDys (ALSnoDys $30.0 \pm 7.4$; ALSDys $25.7 \pm 8.0$; 

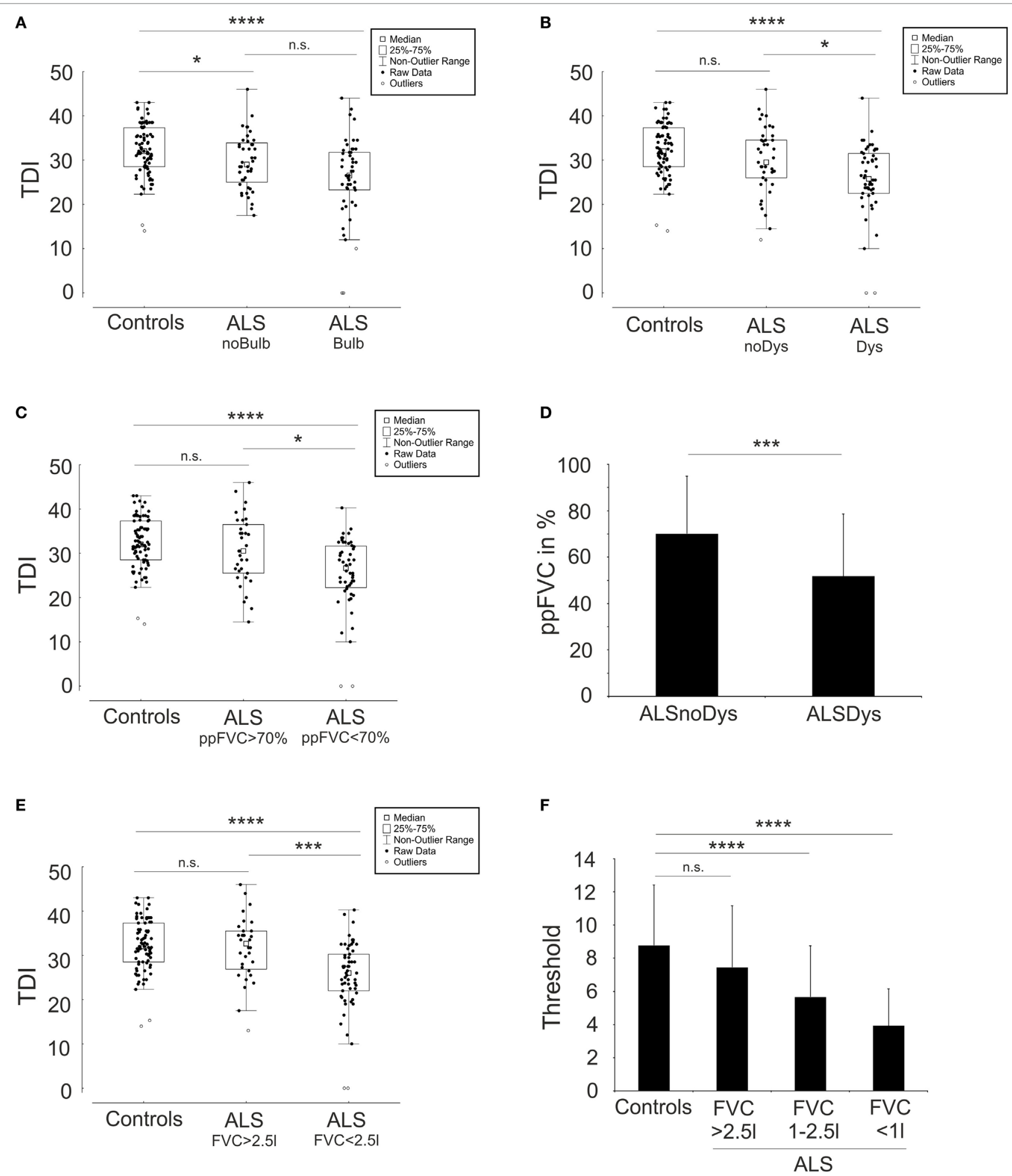

FIGURE 3 | Olfactory dysfunction is a consequence of respiratory dysfunction. Depicted are box plots with single values (dots) concerning the comparison of the composite of the three subtests (TDI) scores of the "Sniffin Sticks" test results between amyotrophic lateral sclerosis (ALS) patients with (Bulb) and without (noBulb) bulbar symptoms (A), the comparison between ALS patients with (Dys) and without (noDys) dyspnea (B), the comparison between ALS patients with $>70 \%$ and $<70 \%$ forced vital capacity of predicted (ppFVC) (C), and the comparison between ALS patients with $>2.5 \mathrm{l}$ and $<2.5 \mathrm{l}$ forced vital capacity (FVC) (E). (D) shows the difference of ppFVC between ALS patients with (ALSDys) and without (ALSnoDys) dyspnea. In (F), the decline of the subtest threshold "T" of the "Sniffin Sticks" test is depicted in comparison to FVC. ${ }^{* \star * *} p<0.0001,{ }^{* \star *} p<0.001,{ }^{* *} p<0.01,{ }^{\star} p<0.05$, not significant (n.s.). 
TABLE 2 | Correlations of demographic and clinical characteristics to "Sniffin Sticks" test results in the ALS study group.

\begin{tabular}{|c|c|c|c|c|}
\hline & TDI score & Threshold & Discrimination & Identification \\
\hline Age & $-0.59 ; N=94^{\star \star \star \star}$ & $-0.30 ; N=94^{\star \star}$ & $-0.42 ; N=94^{\star \star \star \star}$ & $-0.63 ; N=93^{\star \star \star \star}$ \\
\hline Height & n.s.; $N=91$ & n.s.; $N=91$ & n.s.; $N=91$ & n.s.; $N=91$ \\
\hline Weight & $0.31 ; N=93^{\star *}$ & n.s.; $N=93$ & $0.26 ; N=93^{\star}$ & $0.27 ; N=93^{\star \star}$ \\
\hline BMl & $0.28 ; N=90^{\star \star}$ & n.s.; $N=90$ & $0.22 ; N=90^{\star}$ & $0.23 ; N=90^{\star}$ \\
\hline FVC & $0.41 ; N=89^{\star \star \star \star}$ & $0.35 ; N=89^{\star \star \star}$ & $0.31 ; N=89^{* *}$ & $0.32 ; N=89^{\star \star}$ \\
\hline ppFVC & $0.25 ; N=86^{\star}$ & $0.31 ; N=86^{\star \star}$ & n.s.; $N=86$ & n.s.; $N=86$ \\
\hline ALSFRS-R & n.s.; $N=85$ & $0.32 ; N=85^{\star \star}$ & n.s.; $N=85$ & n.s.; $N=85$ \\
\hline Only resp subscore & $0.27 ; N=84^{\star}$ & $0.27 ; N=84^{\star}$ & n.s.; $N=84$ & n.s.; $N=84$ \\
\hline Without resp subscore & n.s.; $N=84$ & $0.28 ; N=84^{\star \star}$ & n.s.; $N=84$ & n.s.; $N=84$ \\
\hline ALSFRS-R slope & n.s.; $N=85$ & n.s.; $N=85$ & n.s.; $N=85$ & n.s.; $N=85$ \\
\hline Disease duration & n.s.; $N=94$ & n.s.; $N=94$ & n.s.; $N=94$ & n.s.; $N=94$ \\
\hline RBD-SQ & n.s.; $N=61$ & n.s.; $N=61$ & n.s.; $N=61$ & n.s.; $N=61$ \\
\hline NMSQuest & n.s.; $N=64$ & n.s.; $N=64$ & n.s.; $N=64$ & n.s.; $N=64$ \\
\hline
\end{tabular}

${ }^{* * * *} p<0.0001,{ }^{* * *} p<0.001,{ }^{* *} p<0.01,{ }^{*} p<0.05$; not significant (n.s.).

Controls $32.3 \pm 5.8 ; p<0.00001$ for ALSDys vs Controls and $p=0.26$ for ALSnoDys vs Controls and $\mathrm{p}<0.05$ for ALSDys vs ALSnoDys) (Figure 3B). Although the total "TDI" was highly impaired in ALSDys group, "I" performance was not different in comparison to controls (ALSDys $10.8 \pm 3.5$; Controls $12.0 \pm 2.2$; $p=0.18$ ). ALSDys group showed a slight reduction of " $D$ " performance compared to controls (ALSDys 9.9 \pm 3.6 ; Controls $11.5 \pm 2.1 ; p<0.05$ ) and a strong reduction in " $\mathrm{T}$ " (ALSDys $5.2 \pm 3.3$; Controls $8.77 \pm 3.6 ; p<0.00001)$. ALSnoDys still performed worse in " $T$ " compared to controls but better than the total ALS patient group and only slightly failed significance level to ALSDys group (ALSnoDys $6.9 \pm 3.3$; ALSDys $5.2 \pm 3.3$; Controls $8.77 \pm 3.6$; for ALSnoDys vs Controls; $p<0.05$ and for ALSnoDys vs ALSDys; $p=0.06$ ). Of note, patients in the ALSnoDys group still had a reduced mean ppFVC of $70 \%$, and patients in the ALSDys group already had relevant involvement of respiratory function with a mean ppFVC of 50\% (Figure 3D). Dyspnea was a subjective parameter and might not represent objective respiratory function.

\section{Respiratory Function Correlates with Impairment of Olfactory Function}

To further evaluate clinical parameters that may correlate with the impairment of olfactory function, we performed several Spearman rank correlations (Table 2). There is a moderate correlation to age as already known from healthy people (46). Additionally weak to moderate correlations to weight and BMI were found for all "Sniffin Sticks" test parameters excluding "T." No correlations were found to ALSFRS-R slope, NMSQuest, RBD-SQ, height, and disease duration. However, we found a highly significant correlation to FVC for all olfactory test parameters. Correlation to ppFVC showed a moderate correlation to "T" and a weak correlation to "TDI." As expected, "D" and "I" did not correlate to ppFVC and were not altered in comparison to controls anyway. Although ppFVC was correlated moderately to ALSFRS-R (rho $=0.54, p<0.00001$ ) even when excluding the respiratory part ( $r h o=0.49, p<0.00001$ ), we found no correlation of "TDI," "I," or "D" to ALSFRS-R. However, there was a weak correlation of " $T$ " to ALSFRS-R even when excluding the respiratory part. Taken together, we suggest that the respiratory function, probably the ability to sniff, was a prominent causal factor of the anyhow-weak impairment of olfactory function in ALS patients.

\section{Decline of Respiratory Function Was Associated with Worse Olfactory Performance}

To further prove the assumed association of olfactory decline with respiratory performance, we divided ALS patients into two groups of patients with FVC below (ALSppFVC $<70, N=52$ ) and above $70 \%$ (ALSppFVC $>70, N=34$ ) of their predicted healthy index value. Of note, ppFVC includes an adjustment for age and gender to exclude age- and gender-dependent influences. "TDI" in ALSppFVC $>70$ was similar to controls, but by contrast, it was significantly reduced in ALSppFVC $<70$ (ALSppFVC $>70$, $30.7 \pm 7.66$; ALSppFVC <70, $25.5 \pm 8.0$; Controls $32.3 \pm 5.8$; $p=0.62$ for ALSppFVC $>70$ vs Controls, $p<0.00001$ for ALSppFVC $<70$ vs Controls, $p<0.05$ for ALSppFVC $>70$ vs ALSppFVC <70) (Figure 3C). The same results were found concerning the total FVC (Figures 3E,F). Although there was a highly significant reduction in "TDI" in the ALSppFVC $<70$ group compared to controls, "I" performance did not differ from controls (ALSppFVC <70, $10.7 \pm 3.6$; Controls $12.0 \pm 2.2$; $p=0.16)$. "D" and "T" performances in ALSppFVC $<70$ group were significantly worse in comparison to controls and to ALSppFVC > 70 ("D":ALSppFVC $>70,11.7 \pm 3.1$;ALSppFVC $<70$, $9.8 \pm 3.4$; Controls $11.5 \pm 2.1 ; p<0.05$ for ALSppFVC $<70$ vs ALSppFVC $>70$ and $p<0.01$ for ALSppFVC $<70$ vs Controls) (“T”: ALSppFVC >70, $7.15 \pm 3.7$; ALSppFVC <70, $5.2 \pm 3.2$; Controls $8.77 \pm 3.6 ; p<0.00001$ for ALSppFVC $<70$ vs Controls and $p<0.05$ for ALSppFVC $<70 \%$ vs ALSppFVC $>70 \%)$. Patients in ALSppFVC $>70$ did not significantly differ from the control group in either "TDI," “T," "D," or "I" performance.

\section{DISCUSSION AND CONCLUSION}

Olfactory dysfunction is very common in neurodegenerative diseases, and in PD, hyposmia can precede motor symptoms for years 
$(25,26)$. The significance of NMS in patients suffering from ALS and its variants is not fully elucidated yet, but scientific evidence is increasing (12). Aside from a handful of publications concerning olfactory function, we recently reported hints for olfactory dysfunction in patients suffering from ALS and its variants (24, 29-33). In the current study, we analyzed olfactory function in ALS patients with the "Sniffin' Sticks" $(45,46)$, a testing device used in clinical routine. In line with previous publications, we found a mild but significant decrease of olfactory performance compared to an age- and sex-matched control group. It is not yet known whether olfactory impairment in ALS patients and its variants is a consequence of the disease spreading in the brain with neurodegeneration in the olfactory system. We did not find differences between patients with a prominent impairment of the lower MNs and those with a prominent impairment of the upper MNs. Therefore, we cannot conclude easily that the mild olfactory impairment is predominantly a result of the disease spreading from the upper MNs.

Previously published work hypothesized that only patients suffering from bulbar symptoms present with olfactory impairment maybe as a consequence of a nonolfactory mechanical problem caused by bulbar palsy (33), but in our study, a significant lowering of olfactory performance was also evident in NoBulb. Thus, the hypothesis that hyposmia is a special symptom of patients with bulbar palsy should be further evaluated. Nasal airway ventilation, especially sniffing performance, is an important prerequisite to maximize odor performance (38). Thus, odor performance depends on sniffing performance and especially on the sniff volume $(38,41)$. Disease progression of ALS and its variants ends in the denervation of the respiratory apparatus, and some patients show a decline of vital capacity already in early disease stages. A recently published work showed a continuous decline of FVC in ALS patients prior to indication for noninvasive ventilation and provided evidence that sniff nasal inspiratory pressure decline was even much stronger (42). This is in agreement with our study showing an association between olfactory impairment and respiratory decline. ALSnoDys or with FVC above $70 \%$ of their predicted healthy index value (ppFVC) performed similar to controls. By contrast, only patients with dyspnea or with ppFVC below $70 \%$ had a worse olfactory performance than controls. Although patients with ppFVC below $70 \%$ had a reduction in general olfactory function, no significant reduction of odor identification performance was found. In these patients, the most prominent impaired parameter was olfactory threshold. As threshold testing yields the concentration above which an odor is perceived, test concentrations are very low in comparison with discrimination and identification being suprathreshold tests (49). This fact suggests that in the latter tests, odor concentrations are still high enough in the olfactory cleft, also in the case of a bad sniffing performance. It might also suggest that the transmission and processing of odors is not altered to a relevant extent in patients suffering from ALS and its variants even if respiratory function is declined. ALS-FRS-R, a score for severity and progression of ALS disease, was associated with a decline of respiratory function. "TDI," "D," and "I" showed no correlation to ALS-FRS-R; however, there was a weak correlation of " $\mathrm{T}$ " to ALS-FRS-R score even when excluding the respiratory part.

Our results do not support an early, clinical relevant neurodegeneration of brain regions acting for the transmission and processing of odors in patients suffering from ALS and its variants. Neuropathological studies in sporadic forms of ALS hypothesize a disease spread starting from motor cortex to effector regions and if ever involve olfactory transmission and processing systems very late in disease progression (21). By contrast, disease spreading or transmission in PD is thought to start in nonmotor regions, especially in the olfactory system (27). In line with that, PD patients perform relatively well in odor threshold but poorly in odor discrimination and identification which mainly depends on the transmission and processing of odor information in the brain, thus hinting toward primary neuronal dysfunction/ degeneration (49).

However, we cannot fully exclude that the clinically relevant reduction of olfactory performance due to neurodegeneration occurs in patients suffering from ALS and its variants in advanced disease stages in which the examination would be very difficult with conventional odor-testing methods. Further neuropathological and MRI-based studies of olfactory bulb and olfactoryprocessing regions may help to further study neurodegeneration in the olfactory brain system in patients of ALS and its variants. Also, prospective studies are warranted to further evaluate the association and impact of olfactory function with/on respiratory function in ALS patients.

In summary, the reduction of olfactory performance in ALS and its variants should be considered as a consequence of impairment of respiratory function, and olfactory threshold testing might be an early marker of respiratory decline in ALS. However, because of the case-control study design with its limitations, further prospective studies of odor threshold testing in combination with multiple cardiorespiratory parameters such as vital capacity, sniffing capacity, and diaphragm ultrasound are needed to estimate its significance and diagnostic potential in patients suffering from ALS and its variants.

\section{ETHICS STATEMENT}

The study was approved by the institutional review board at the Technische Universität Dresden (EK 393122012, EK 49022016), and patients gave their informed consent.

\section{AUTHOR CONTRIBUTIONS}

RG: Conception and design, collection and assembly of data, data analysis and interpretation, manuscript drafting. WS, AH, TH, MW, and AS: Collection and/or assembly of data, critical revision of manuscript. AH: Conception and design, principal investigator, collection and assembly of data, data analysis and interpretation, manuscript drafting.

\section{ACKNOWLEDGMENTS}

We thank all the patients who participated in this study. 


\section{REFERENCES}

1. Kim WK, Liu X, Sandner J, Pasmantier M, Andrews J, Rowland LP, et al. Study of 962 patients indicates progressive muscular atrophy is a form of ALS. Neurology (2009) 73(20):1686-92. doi:10.1212/WNL. 0b013e3181c1dea3

2. Wais V, Rosenbohm A, Petri S, Kollewe K, Hermann A, Storch A, et al. The concept and diagnostic criteria of primary lateral sclerosis. Acta Neurol Scand (2017) 136(3):204-11. doi:10.1111/ane.12713

3. Eisen A, Braak H, Tredici KD, Lemon R, Ludolph AC, Kiernan MC. Cortical influences drive amyotrophic lateral sclerosis. J Neurol Neurosurg Psychiatry (2017) 88:917-24. doi:10.1136/jnnp-2017-315573

4. Brundin P, Melki R, Kopito R. Prion-like transmission of protein aggregates in neurodegenerative diseases. Nat Rev Mol Cell Biol (2010) 11(4):301-7. doi:10.1038/nrm 2873

5. Chaudhuri KR, Martinez-Martin P, Schapira AH, Stocchi F, Sethi K, Odin P, et al. International multicenter pilot study of the first comprehensive self-completed nonmotor symptoms questionnaire for Parkinson's disease: the NMSQuest study. Mov Disord (2006) 21(7):916-23. doi:10.1002/mds. 20844

6. Chaudhuri KR, Martinez-Martin P, Brown RG, Sethi K, Stocchi F, Odin P, et al. The metric properties of a novel non-motor symptoms scale for Parkinson's disease: results from an international pilot study. Mov Disord (2007) 22(13):1901-11. doi:10.1002/mds.21596

7. Phukan J, Pender NP, Hardiman O. Cognitive impairment in amyotrophic lateral sclerosis. Lancet Neurol (2007) 6(11):994-1003. doi:10.1016/s14744422(07)70265-x

8. Beeldman E, Raaphorst J, Klein Twennaar M, de Visser M, Schmand BA, de Haan RJ. The cognitive profile of ALS: a systematic review and metaanalysis update. JNeurol Neurosurg Psychiatry (2016) 87(6):611-9. doi:10.1136/jnnp-2015-310734

9. Burrell JR, Halliday GM, Kril JJ, Ittner LM, Götz J, Kiernan MC, et al. The frontotemporal dementia-motor neuron disease continuum. Lancet (2016) 388(10047):919-31. doi:10.1016/s0140-6736(16)00737-6

10. Rabkin J, Goetz R, Murphy JM, Factor-Litvak P, Mitsumoto H; ALS COSMOS Study Group. Cognitive impairment, behavioral impairment, depression, and wish to die in an ALS cohort. Neurology (2016) 87(13):1320-8. doi:10.1212/wnl.0000000000003035

11. Mackenzie IR, Feldman H. Extrapyramidal features in patients with motor neuron disease and dementia; a clinicopathological correlative study. Acta Neuropathol (2004) 107(4):336-40. doi:10.1007/s00401-003-0814-y

12. Swinnen B, Robberecht W. The phenotypic variability of amyotrophic lateral sclerosis. Nat Rev Neurol (2014) 10(11):661-70. doi:10.1038/nrneurol. 2014.184

13. Toepfer M, Folwaczny C, Klauser A, Riepl RL, Muller-Felber W, Pongratz D. Gastrointestinal dysfunction in amyotrophic lateral sclerosis. Amyotroph Lateral Scler Other Motor Neuron Disord (1999) 1(1):15-9. doi:10.1080/ 146608299300079484

14. Baltadzhieva R, Gurevich T, Korczyn AD. Autonomic impairment in amyotrophic lateral sclerosis. Curr Opin Neurol (2005) 18(5):487-93. doi:10.1097/ 01.wco.0000183114.76056.0e

15. Pavlovic S, Stevic Z, Milovanovic B, Milicic B, Rakocevic-Stojanovic V, Lavrnic D, et al. Impairment of cardiac autonomic control in patients with amyotrophic lateral sclerosis. Amyotroph Lateral Scler (2010) 11(3):272-6. doi:10.3109/17482960903390855

16. Nubling GS, Mie E, Bauer RM, Hensler M, Lorenzl S, Hapfelmeier A, et al. Increased prevalence of bladder and intestinal dysfunction in amyotrophic lateral sclerosis. Amyotroph Lateral Scler Frontotemporal Degener (2014) 15(3-4):174-9. doi:10.3109/21678421.2013.868001

17. Arlandis S, Vazquez-Costa JF, Martinez-Cuenca E, Sevilla T, Boronat F, Broseta E. Urodynamic findings in amyotrophic lateral sclerosis patients with lower urinary tract symptoms: results from a pilot study. Neurourol Urodyn (2017) 36(3):626-31. doi:10.1002/nau.22976

18. Truini A, Biasiotta A, Onesti E, Di Stefano G, Ceccanti M, La Cesa S, et al. Small-fibre neuropathy related to bulbar and spinal-onset in patients with ALS. J Neurol (2015) 262(4):1014-8. doi:10.1007/s00415-015-7672-0

19. Desai J, Swash M. Extrapyramidal involvement in amyotrophic lateral sclerosis: backward falls and retropulsion. J Neurol Neurosurg Psychiatry (1999) 67(2):214-6. doi:10.1136/jnnp.67.2.214
20. Manno C, Lipari A, Bono V, Taiello AC, La Bella V. Sporadic Parkinson disease and amyotrophic lateral sclerosis complex (Brait-Fahn-Schwartz disease). J Neurol Sci (2013) 326(1-2):104-6. doi:10.1016/j.jns.2013.01.009

21. Braak H, Brettschneider J, Ludolph AC, Lee VM, Trojanowski JQ, Del Tredici K. Amyotrophic lateral sclerosis-a model of corticofugal axonal spread. Nat Rev Neurol (2013) 9(12):708-14. doi:10.1038/nrneurol.2013.221

22. Brettschneider J, Del Tredici K, Toledo JB, Robinson JL, Irwin DJ, Grossman M, et al. Stages of pTDP-43 pathology in amyotrophic lateral sclerosis. Ann Neurol (2013) 74(1):20-38. doi:10.1002/ana.23937

23. Hermann A, Reuner U, Schaefer J, Fathinia P, Leimert T, Kassubek J, et al. The diagnostic value of midbrain hyperechogenicity in ALS is limited for discriminating key ALS differential diagnoses. BMC Neurol (2015) 15:33. doi:10.1186/s12883-015-0280-x

24. Gunther R, Richter N, Sauerbier A, Chaudhuri KR, Martinez-Martin P, Storch A, et al. Non-motor symptoms in patients suffering from motor neuron diseases. Front Neurol (2016) 7:117. doi:10.3389/fneur.2016.00117

25. Ross GW, Petrovitch H, Abbott RD, Tanner CM, Popper J, Masaki K, et al. Association of olfactory dysfunction with risk for future Parkinson's disease. Ann Neurol (2008) 63(2):167-73. doi:10.1002/ana.21291

26. Reichmann H. Premotor diagnosis of Parkinson's disease. Neurosci Bull (2017) 33(5):526-34. doi:10.1007/s12264-017-0159-5

27. Braak H, Bohl JR, Muller CM, Rub U, de Vos RA, Del Tredici K. Stanley Fahn Lecture 2005: the staging procedure for the inclusion body pathology associated with sporadic Parkinson's disease reconsidered. Mov Disord (2006) 21(12):2042-51. doi:10.1002/mds.21065

28. Xiao Q, Chen S, Le W. Hyposmia: a possible biomarker of Parkinson's disease. Neurosci Bull (2014) 30(1):134-40. doi:10.1007/s12264-013-1390-3

29. Elian M. Olfactory impairment in motor neuron disease: a pilot study. J Neurol Neurosurg Psychiatry (1991) 54(10):927-8. doi:10.1136/jnnp.54.10.927

30. Doty RL. Studies of olfactory dysfunction in major neurological disorders. Adv Biosci (1994) 93:593-602.

31. Sajjadian A, Doty RL, Gutnick DN, Chirurgi RJ, Sivak M, Perl D, et al. Olfactory dysfunction in amyotrophic lateral sclerosis. Neurodegeneration (1994) 3(2):153-7.

32. Ahlskog JE, Waring SC, Petersen RC, Esteban-Santillan C, Craig UK, O'Brien PC, et al. Olfactory dysfunction in Guamanian ALS, parkinsonism, and dementia. Neurology (1998) 51(6):1672-7. doi:10.1212/WNL.51.6.1672

33. Hawkes CH, Shephard BC, Geddes JF, Body GD, Martin JE. Olfactory disorder in motor neuron disease. Exp Neurol (1998) 150(2):248-53. doi:10.1006/ exnr.1997.6773

34. Lang CJ, Schwandner K, Hecht M. Do patients with motor neuron disease suffer from disorders of taste or smell? Amyotroph Lateral Scler (2011) 12(5):368-71. doi:10.3109/17482968.2011.579133

35. Pilotto A, Rossi F, Rinaldi F, Compostella S, Cosseddu M, Borroni B, et al. Exploring olfactory function and its relation with behavioral and cognitive impairment in amyotrophic lateral sclerosis patients: a cross-sectional study. Neurodegener Dis (2016) 16(5-6):411-6. doi:10.1159/000446802

36. Takeda T, Iijima M, Uchihara T, Ohashi T, Seilhean D, Duyckaerts C, et al. TDP-43 pathology progression along the olfactory pathway as a possible substrate for olfactory impairment in amyotrophic lateral sclerosis. J Neuropathol Exp Neurol (2015) 74(6):547-56. doi:10.1097/nen.0000000000000198

37. Damm M, Temmel A, Welge-Lussen A, Eckel HE, Kreft MP, Klussmann JP, et al. [Olfactory dysfunctions. Epidemiology and therapy in Germany, Austria and Switzerland]. HNO (2004) 52(2):112-20. doi:10.1007/s00106-003-0877-z

38. Tucker D. Physical variables in the olfactory stimulation process. JGen Physiol (1963) 46:453-89. doi:10.1085/jgp.46.3.453

39. Sobel N, Khan RM, Hartley CA, Sullivan EV, Gabrieli JD. Sniffing longer rather than stronger to maintain olfactory detection threshold. Chem Senses (2000) 25(1):1-8. doi:10.1093/chemse/25.1.1

40. Sobel N, Thomason ME, Stappen I, Tanner CM, Tetrud JW, Bower JM, et al. An impairment in sniffing contributes to the olfactory impairment in Parkinson's disease. Proc Natl Acad Sci U S A (2001) 98(7):4154-9. doi:10.1073/ pnas.071061598

41. Laing DG. Natural sniffing gives optimum odour perception for humans Perception (1983) 12(2):99-117. doi:10.1068/p120099

42. Tilanus TBM, Groothuis JT, TenBroek-Pastoor JMC, Feuth TB, Heijdra YF, Slenders JPL, et al. The predictive value of respiratory function tests for non-invasive ventilation in amyotrophic lateral sclerosis. Respir Res (2017) 18(1):144. doi:10.1186/s12931-017-0624-8 
43. Brooks BR. El Escorial World Federation of Neurology criteria for the diagnosis of amyotrophic lateral sclerosis. Subcommittee on motor neuron diseases/amyotrophic lateral sclerosis of the World Federation of Neurology Research Group on Neuromuscular Diseases and the El Escorial "Clinical limits of amyotrophic lateral sclerosis" workshop contributors. J Neurol Sci (1994) 124(Suppl):96-107.

44. Brooks BR, Miller RG, Swash M, Munsat TL. El Escorial revisited: revised criteria for the diagnosis of amyotrophic lateral sclerosis. Amyotroph Lateral Scler Other Motor Neuron Disord (2000) 1(5):293-9. doi:10.1080/ 146608200300079536

45. Hummel T, Sekinger B, Wolf SR, Pauli E, Kobal G. 'Sniffin' Sticks': olfactory performance assessed by the combined testing of odor identification, odor discrimination and olfactory threshold. Chem Senses (1997) 22(1):39-52. doi:10.1093/chemse/22.1.39

46. Hummel T, Kobal G, Gudziol H, Mackay-Sim A. Normative data for the "Sniffin' Sticks" including tests of odor identification, odor discrimination, and olfactory thresholds: an upgrade based on a group of more than 3,000 subjects. Eur Arch Otorhinolaryngol (2007) 264(3):237-43. doi:10.1007/ s00405-006-0173-0

47. Quanjer PH, Stanojevic S, Cole TJ, Baur X, Hall GL, Culver BH, et al. Multi-ethnic reference values for spirometry for the 3-95-yr age range: the global lung function 2012 equations. Eur Respir J (2012) 40(6):1324-43. doi:10.1183/09031936.00080312

48. Criee CP, Baur X, Berdel D, Bosch D, Gappa M, Haidl P, et al. [Standardization of spirometry: 2015 update. Published by German Atemwegsliga, German Respiratory Society and German Society of Occupational and Environmental Medicine]. Pneumologie (2015) 69(3):147-64. doi:10.1055/s-0034-1391345

49. Whitcroft KL, Cuevas M, Haehner A, Hummel T. Patterns of olfactory impairment reflect underlying disease etiology. Laryngoscope (2017) 127(2): 291-5. doi:10.1002/lary.26229

Conflict of Interest Statement: The authors declare that the research was conducted in the absence of any commercial or financial relationships that could be construed as a potential conflict of interest.

Copyright (C) 2018 Günther, Schrempf, Hähner, Hummel, Wolz, Storch and Hermann. This is an open-access article distributed under the terms of the Creative Commons Attribution License (CC BY). The use, distribution or reproduction in other forums is permitted, provided the original author(s) and the copyright owner are credited and that the original publication in this journal is cited, in accordance with accepted academic practice. No use, distribution or reproduction is permitted which does not comply with these terms. 